\title{
Integrity of cerebral white matter in type 1 diabetes
}

\author{
A. M. Wessels
}

Received: 21 March 2008 / Accepted: 28 March 2008 / Published online: 1 May 2008

(C) Springer-Verlag 2008

Keywords Brain structure - Type 1 diabetes .

White matter volume $\cdot$ White matter hyperintensities

\section{Abbreviation \\ WMHs White matter hyperintensities}

To the Editor: In a recent issue of Diabetologia, Weinger et al. [1] report evidence of only mild white matter hyperintensities (WMHs) in a sample of adults with type 1 diabetes. These WMHs were comparable to those seen in a demographically similar sample of non-diabetic adults, and no association between diabetes characteristics and WMHs was found.

As stated by the authors and from what is known from the literature, there is no clear understanding of the clinical relevance of WMHs. However there is evidence to suggest that whole grey and white matter volume, as compared with lesion burden, is more closely related to neuropsychological performance and neuropsychiatric symptoms $[2,3]$. Our investigations of total white and grey matter volume in patients with type 1 diabetes revealed a relationship between cerebral white matter volume and cognitive performance [4]. Patients with diabetes demonstrated several subtle neuropsychological deficits, and patients with a microvascular complication (defined as proliferative retinopathy) showed a decreased white matter volume that

A. M. Wessels $(\bowtie)$

IU Center for Neuroimaging,

Division of Imaging Sciences, Department of Radiology,

950 W. Walnut Street R2 E159,

Indianapolis, IN 46202, USA

e-mail: awessels@iupui.edu was associated with worse cognitive performance. This suggests that cognitive decline is at least partly mediated by microvascular disease.

Despite the fact that these studies differ methodologically and focus on different aspects of the brain (i.e. WMHs vs white matter volume), both aimed to elucidate the underlying mechanism of cognitive decline in type 1 diabetes. It is therefore unfortunate that Weinger et al. did not include this specific consideration in their paper.

Whether WMHs are associated with white matter volume is still under debate. The results of a study by Wen et al. [5] suggest that WMHs are associated with loss of brain volume, which could presumably be the result of white matter pathology [6]. In contrast, Smith at al. [7] reported that decreased white matter volume measured from magnetic resonance imaging was associated with dementia and with lower cognitive test scores, whereas no such association was found with periventricular WMHs identified on the same images. WMHs were not related to white matter volume, stroke or dementia. Nonetheless, low white matter volume was strongly related to dementia and poor cognitive performance in this sample.

In the elderly, the periventricular WMHs have been shown to be unrelated to total white matter volume loss [8]. Additional data from diffusion-weighted and magnetisation transfer (MT) imaging studies $[9,10]$ reveal that fractional anisotropy and the magnetisation ratio (a measure of the integrity of the microstructure) in white matter significantly decrease with age. These decreases suggest that white matter volume loss may result from these microscopic structural changes, which include myelin and axonal loss, dilatation of perivascular spaces and gliosis.

To understand the mechanisms by which diabetes might affect the brain, it is important to consider the apparent 
discrepancies between the various neuroimaging findings on the one hand, and the cognitive assessments on the other.

The study of Weinger et al. [1] does not include measurements of the volume of the WMHs. This is unfortunate, since the information provided by categorical ratings is of lower sensitivity than that provided by fully quantitative analysis of WMHs, and is therefore less likely to reveal subtle effects (such as those associated with diabetes) on brain structure. It would be of particular interest to assess white matter volume in the sample studied by Weinger et al., and to determine whether a correlation with WMH volume exists. This might clarify the complex underlying pathophysiology of cognitive decline in type 1 diabetes.

Whether diabetes-related cognitive decline is due to grey or white matter disease is still not clear, and future longitudinal studies should assess the integrity of these brain compartments, to enhance our understanding of the potential mechanisms underlying structural brain changes and their effect on cognitive performance in patients with type 1 diabetes.

Duality of interest The author declares that there is no duality of interest associated with this manuscript.

\section{References}

1. Weinger K, Jacobson AM, Musen G et al (2008) The effects of type 1 diabetes on cerebral white matter. Diabetologia 51:417-425
2. Benedict RH, Carone DA, Bakshi R (2004) Correlating brain atrophy with cognitive dysfunction, mood disturbances, and personality disorder in multiple sclerosis. J Neuroimaging $14: 36 \mathrm{~S}-45 \mathrm{~S}$

3. Benedict RH, Weinstock-Guttman B, Fishman I, Sharma J, Tjoa CW, Bakshi R (2004) Prediction of neuropsychological impairment in multiple sclerosis: comparison of conventional magnetic resonance imaging measures of atrophy and lesion burden. Arch Neurol 61:226-230

4. Wessels AM, Rombouts SA, Remijnse PL et al (2007) Cognitive performance in type 1 diabetes patients is associated with cerebral white matter volume. Diabetologia 50:1763-1769

5. Wen W, Sachdev PS, Chen X, Anstey K (2006) Gray matter reduction is correlated with white matter hyperintensity volume: a voxel-based morphometric study in a large epidemiological sample. Neuroimage 29:1031-1039

6. DeCarli C, Murphy DG, Tranh M et al (1995) The effect of white matter hyperintensity volume on brain structure, cognitive performance, and cerebral metabolism of glucose in 51 healthy adults. Neurology 45:2077-2084

7. Smith CD, Snowdon DA, Wang H, Markesbery WR (2000) White matter volumes and periventricular white matter hyperintensities in aging and dementia. Neurology 54:838-842

8. Braffman BH, Zimmerman RA, Trojanowski JQ, Gonatas NK, Hickey WF, Schlaepfer WW (1988) Brain MR: pathologic correlation with gross and histopathology. 2. Hyperintense whitematter foci in the elderly. AJR Am J Roentgenol 151:559-566

9. Scheltens P, Barkhof F, Leys D, Wolters EC, Ravid R, Kamphorst W (1995) Histopathologic correlates of white matter changes on MRI in Alzheimer's disease and normal aging. Neurology 45:883-888

10. Virta A, Barnett A, Pierpaoli C (1999) Visualizing and characterizing white matter fiber structure and architecture in the human pyramidal tract using diffusion tensor MRI. Magn Reson Imaging 17:1121-1133 